Supporting Information

for

\title{
Spin Dynamics of the Radicals Produced from Methane Sulfonic Acid and Acetic Acid by Hydroxyl Radicals in Aqueous Solution Studied by Means of Time-Resolved Electron Spin Resonance Spectroscopy.
}

Yutaka Kuwabara, Masahiro Kitazawa, Atsuko Y. Nosaka, and Yoshio Nosaka*

Department of Materials Science and Technology, Nagaoka University of Technology, 1603-1 Kamitomioka, Nagaoka, 940-2188 Japan

This supporting information contains three topics.

1. Effect of the bandwidth of preamplifier on TR-ESR spectra of ethanol radical. (Pages S2-S4)

2. Energy diagram for the 1:2:1 triplet signal in TR-ESR spectra and the effect of CIDEP (Page S5)

3. Additional figures for the TR-ESR spectra of acetic acid (AA) radicals (Pages S6$\mathrm{S} 10)$ 


\section{Effect of the band-width of preamplifier}

The ESR signals contain noises of several frequencies. In order to reduce the noise level for the digital averager, the bandwidth of the preamplifier becomes important. We tried to use a preamplifier of 10-MHz bandwidth characteristics (Model 5307, NF Electronic Instruments, Inc.) and it was compared with the amplifier (SR-560, Stanford Research System) of 1-MHz bandwidth characteristics. The repetition of the pulsed laser was $18 \mathrm{kHz}$ in this experiment. The sample used was aqueous solution of $4 \mathrm{M}$ ethanol and $1 \mathrm{M} \mathrm{H}_{2} \mathrm{O}_{2}$. Figure $\mathrm{S} 1$ shows the time-profile of the ESR signal at the magnetic field of $344.9 \mathrm{mT}$ for the largest peak. And Figure S2 shows the timeresolved ESR spectra obtained by accumulating the time period of $0.16 \mu$ s to $0.6 \mu \mathrm{s}$ after the laser pulse. Though the $\mathrm{S} / \mathrm{N}$ (signal to noise ratio) of S1(a) and S2(a) was poor in comparison to $S 1(b)$ and $S 2(b)$, the response in the time profile shown in $S 1$ (b) was not much different from that in S1(a). This observation indicates that the time response was determined other than the preamplifier, for example the MW unit and the MW cavity. Since the response was not much better than $1 \mathrm{MHz}$, we employed the preamplifier having the bandwidth (LPF characteristics) of $1 \mathrm{MHz}$. 

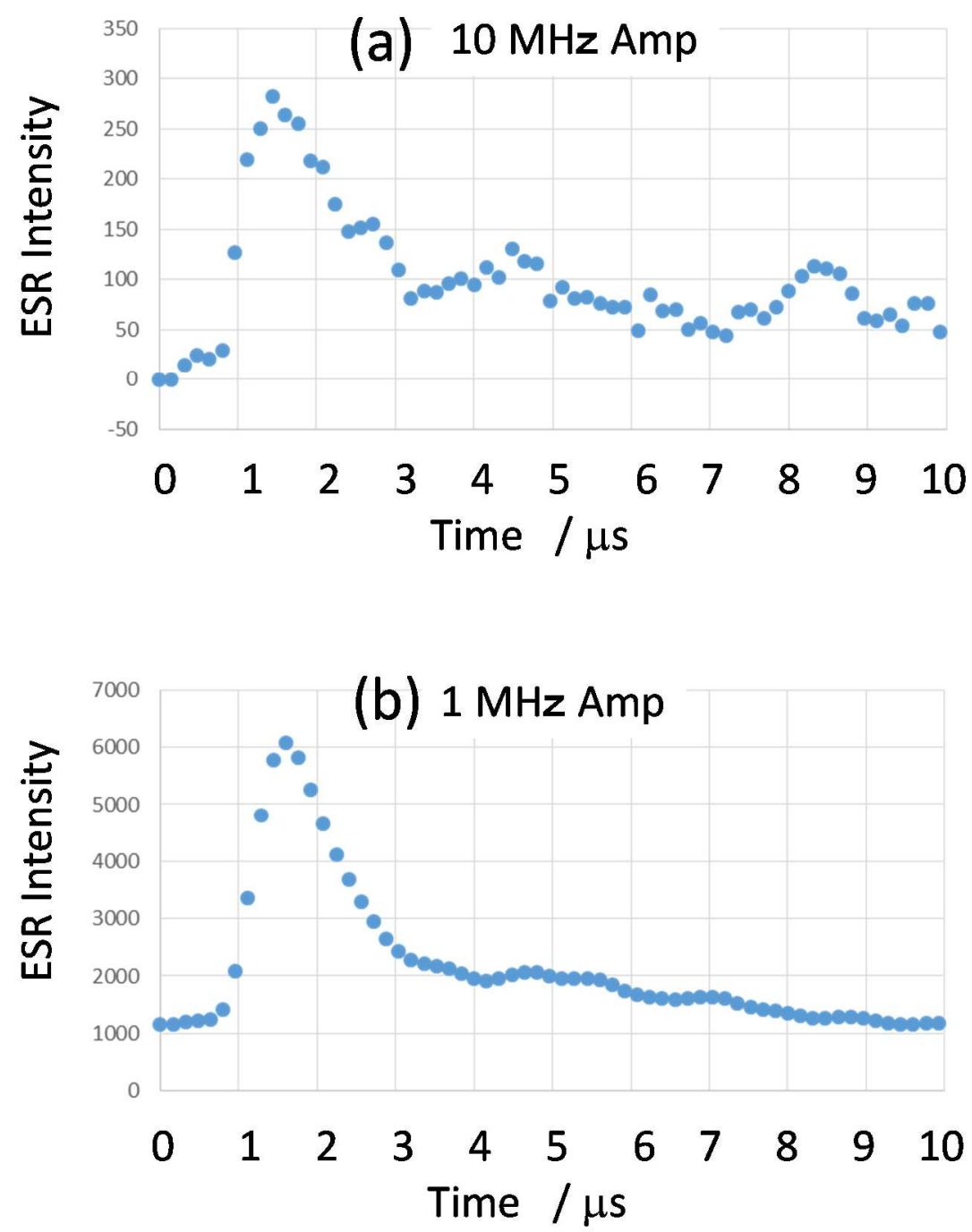

Fig S1 Time-profiles of the ESR signal measured by using preamplifiers of different bandwidth specifications. (a) $10 \mathrm{MHz}$ and (b) $1 \mathrm{MHz}$. 

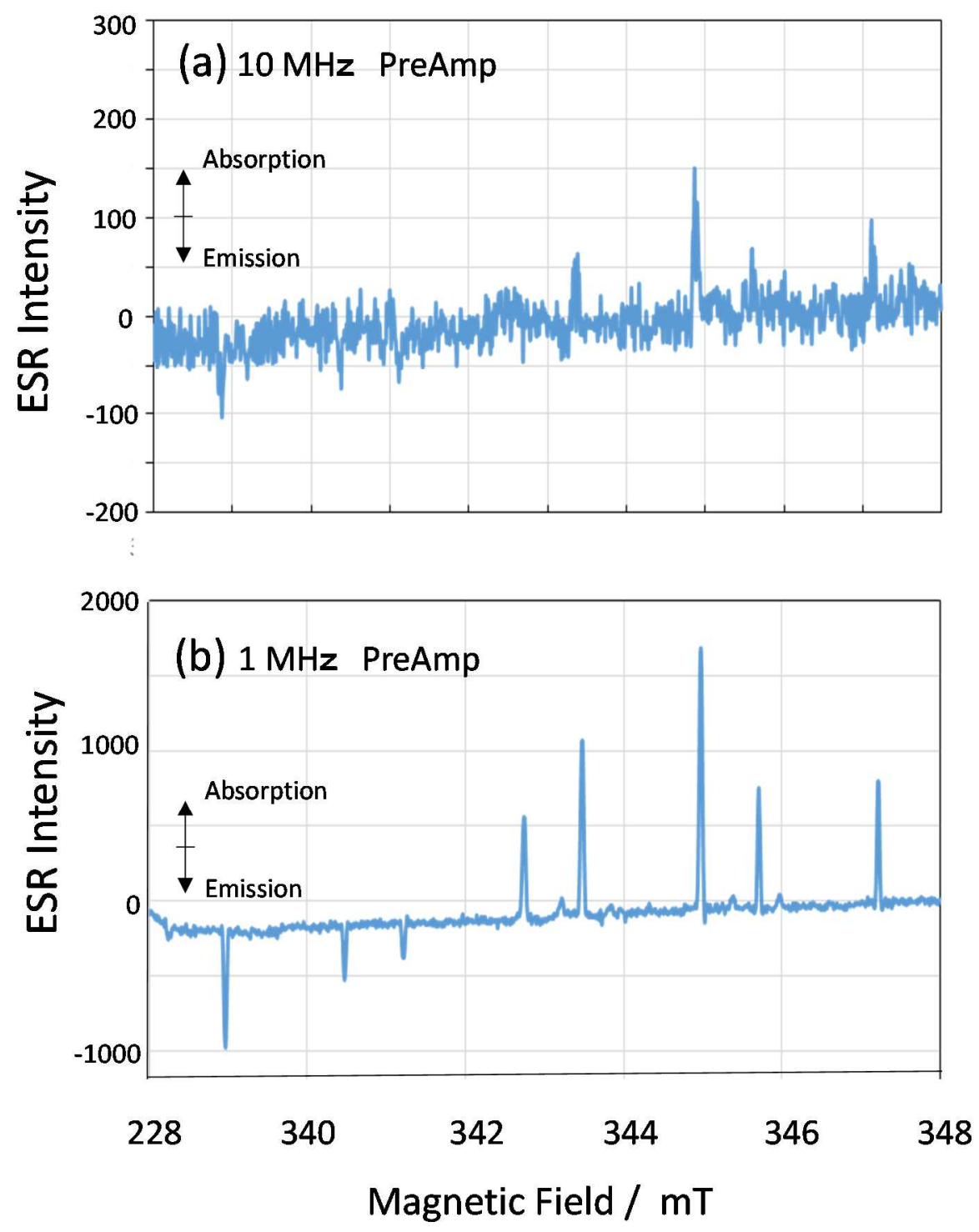

Fig S2 Comparison of the TR-ESR spectra measured for ethanol- $\mathrm{H}_{2} \mathrm{O}_{2}$ solution by using preamplifiers of different bandwidth specifications. (a) $10 \mathrm{MHz}$ and (b) $1 \mathrm{MHz}$. 
2. Energy diagram for the 1:2:1 triplet signal in TR-ESR spectra and the effect of CIDEP

In the usual ESR spectra, the carbon radicals having ${ }^{\bullet} \mathrm{CH}_{2}$ - structure provide 1:2:1 triplet signal. As shown in Figure S3(a), the three peaks in the signal are assigned to the transitions from $m_{\mathrm{s}}=-\frac{1}{2}$ to $m_{\mathrm{s}}=+1 / 2$ in the electron spin states which are modified by the coupled nuclear spin states of $M_{\mathrm{I}}=-1,0$, and +1 for the peaks $\mathrm{L}, \mathrm{C}$, and $\mathrm{H}$, respectively. The ESR signal intensity is proportional to the difference between the populations of two electron spin states, which is caused by the Boltzmann distribution in the thermal equilibrium. By the effect of CIDEP, the number of radicals of the two spin states suddenly becomes equal, and the population difference of each nuclear spin state is changed as shown in Figure 3(b). As the result, the emission signal can be seen for $M_{\mathrm{I}=}=1$, and no signal is observed for $M_{\mathrm{I}=}=0$. Thus, the ratio of the signal intensities in the TR-ESR spectra becomes -1:0:1.

(a)

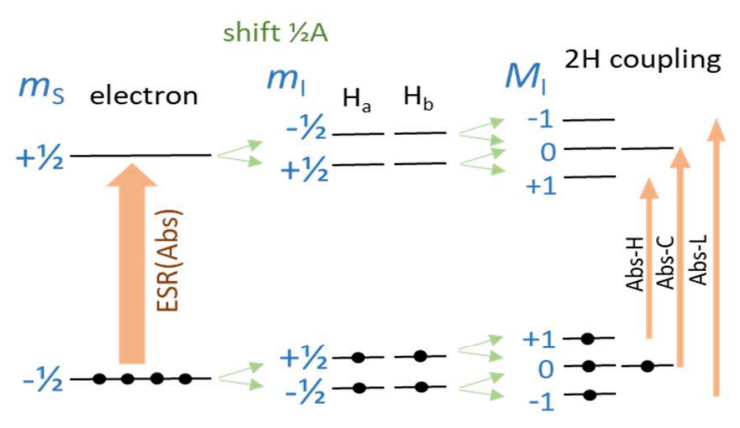

$f=1$ Boltzmann distribution (Thermalized equilibrium)

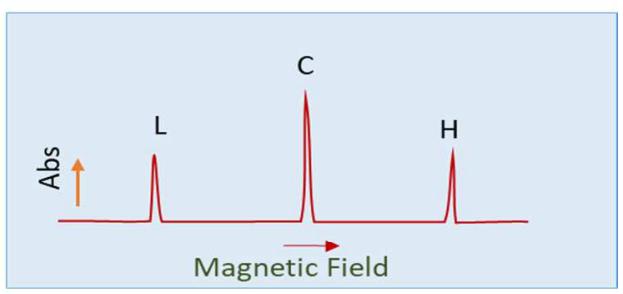

Normal ESR spectrum

(b)
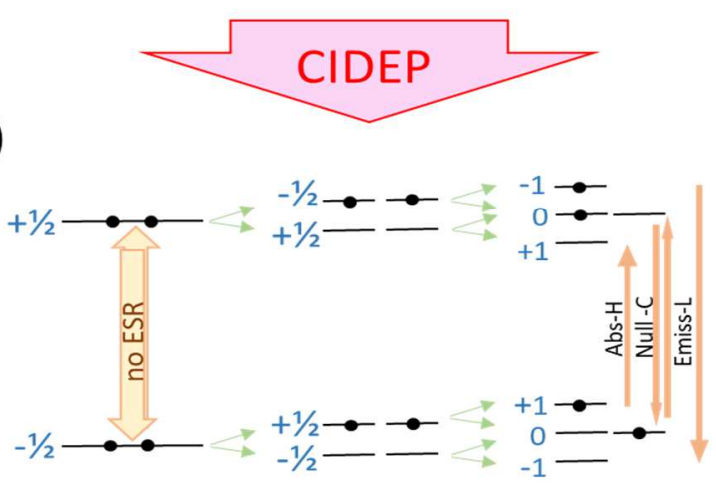

$f=0$ Non thermalized distribution

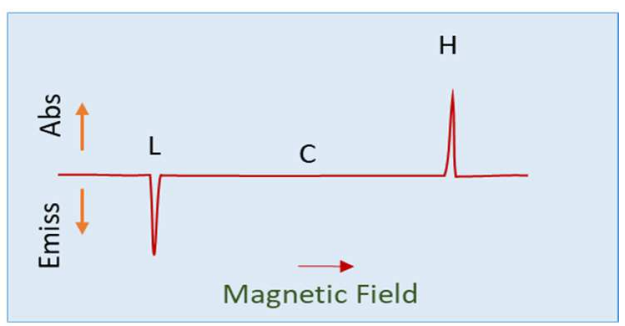

TR-ESR spectrum

Fig S3 Energy level diagrams of $\bullet \mathrm{CH}_{2}$ radical under the magnetic field for (a) normal ESR absorption spectrum of $f=1$ and (b) TR-ESR spectrum of $f=0$ with emission signal by the CIDEP effect. 
3. Additional figures for the TR-ESR spectra of ${ }^{\bullet} \mathrm{CH}_{2} \mathrm{COOH}$ and ${ }^{-} \mathrm{CH}_{2} \mathrm{COO}^{-}$ radicals

Figures S4(a) and (b) show the time profiles of ESR signals of the central peak measured at $\mathrm{pH} 1.0$ and 6.1, respectively. Figure S5 and S6 show the fine structure of the central peak (C) in TR-ESR spectra at pH 2.5 and pH 4.9, respectively. Figure S7and S8 show the fine structure of the low-field peak (L) in TR-ESR spectra at $\mathrm{pH}$ 6.1 .

(a) $\mathrm{pH} 1.0$

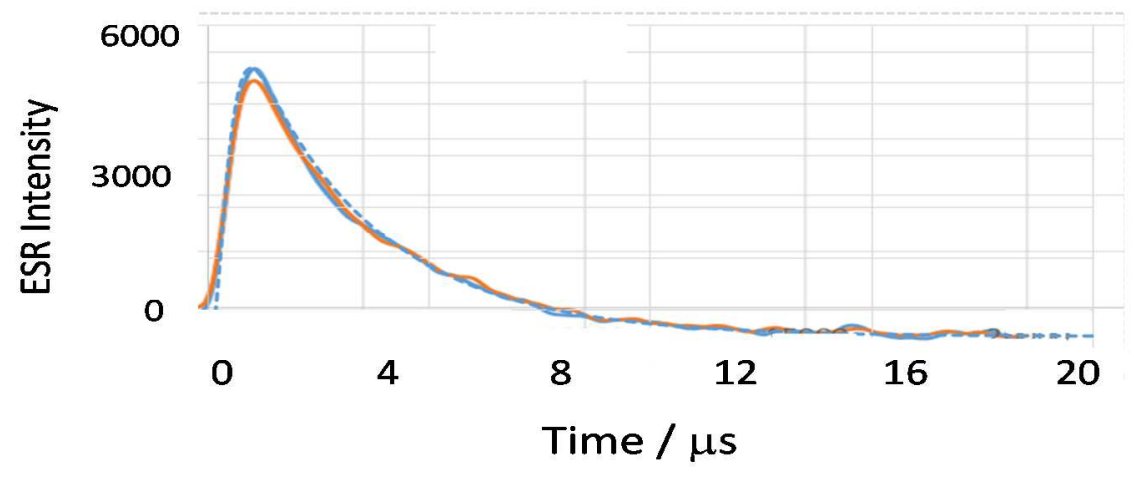

(b) $\mathrm{pH} 6.1$

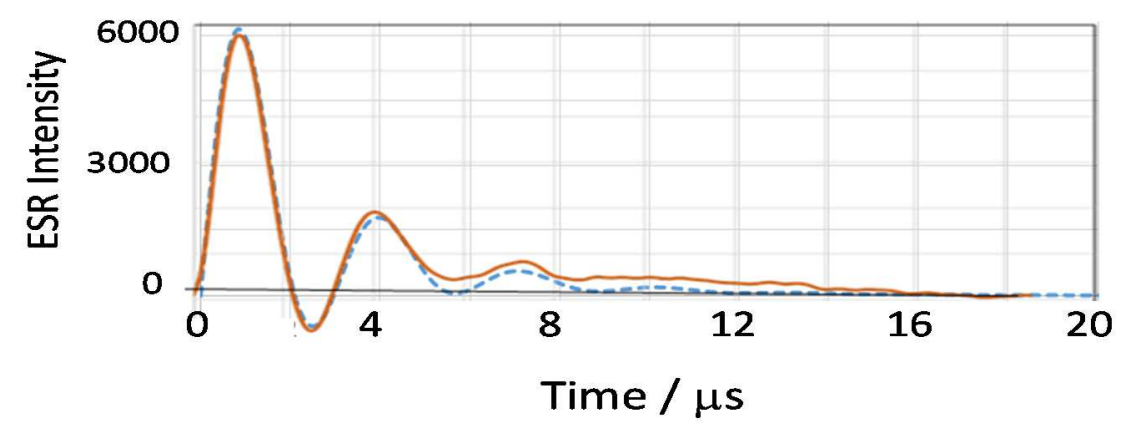

Fig S4 Time profiles of the ESR intensity of the central signal (C) for AA radical after the laser pulse on the solution containing $2 \mathrm{M} \mathrm{AA}$ and $1 \mathrm{M} \mathrm{H}_{2} \mathrm{O}_{2}$ of (a) $\mathrm{pH} 1.0$ and (b) $\mathrm{pH}$ 6.1. Broken lines are calculated with eq (1) adopting the parameters shown in Table 1. 
(a)

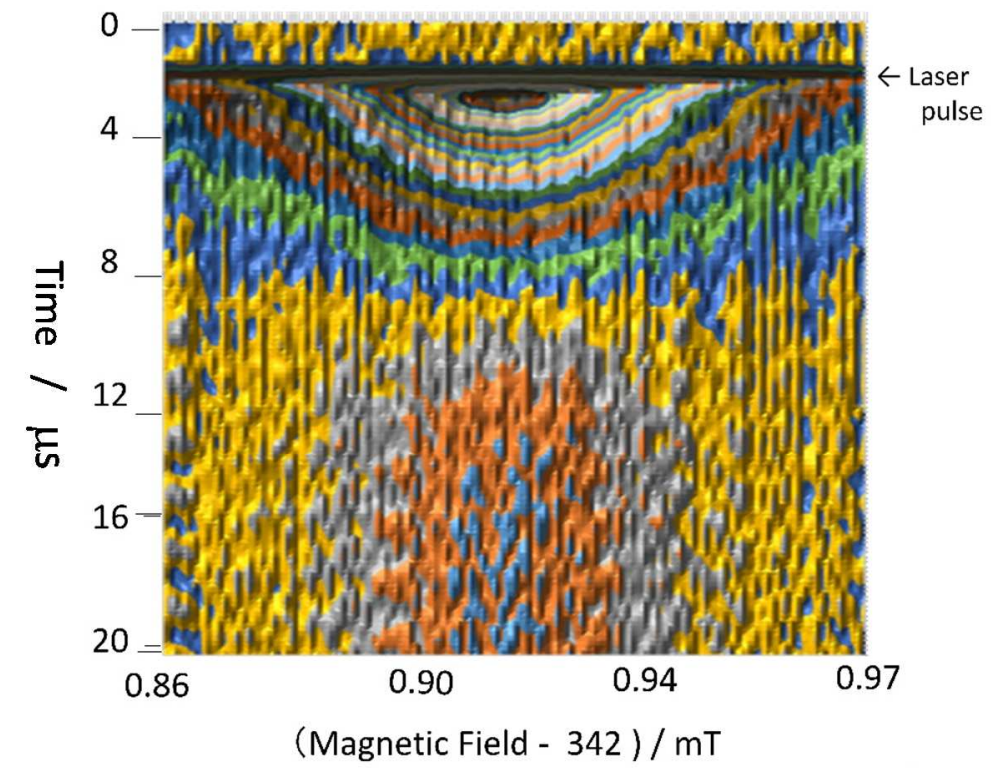

(b)

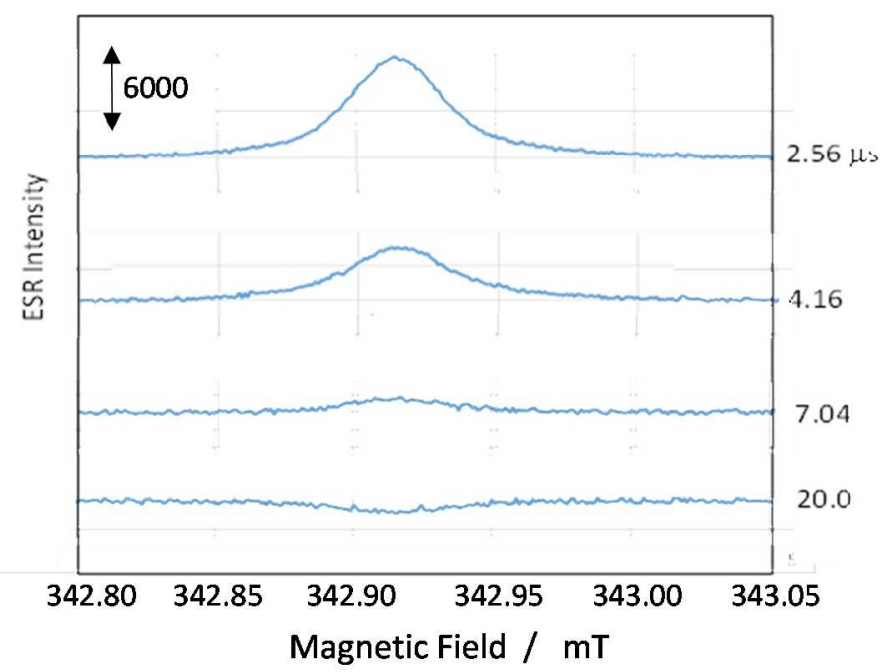

Fig S5 (a) Two dimensional TR-ESR plot as a function of time and magnetic field for the central signal (C) of $\bullet \mathrm{CH}_{2} \mathrm{COOH}$ radical at $\mathrm{pH}$ 2.5. The color changes every 200 in unit of the ESR intensity. (b) Time resolved ESR spectra at the designated times. The time resolution was $0.16 \mu$ s and the magnetic field step was 0.000977 $\mathrm{mT}$. 


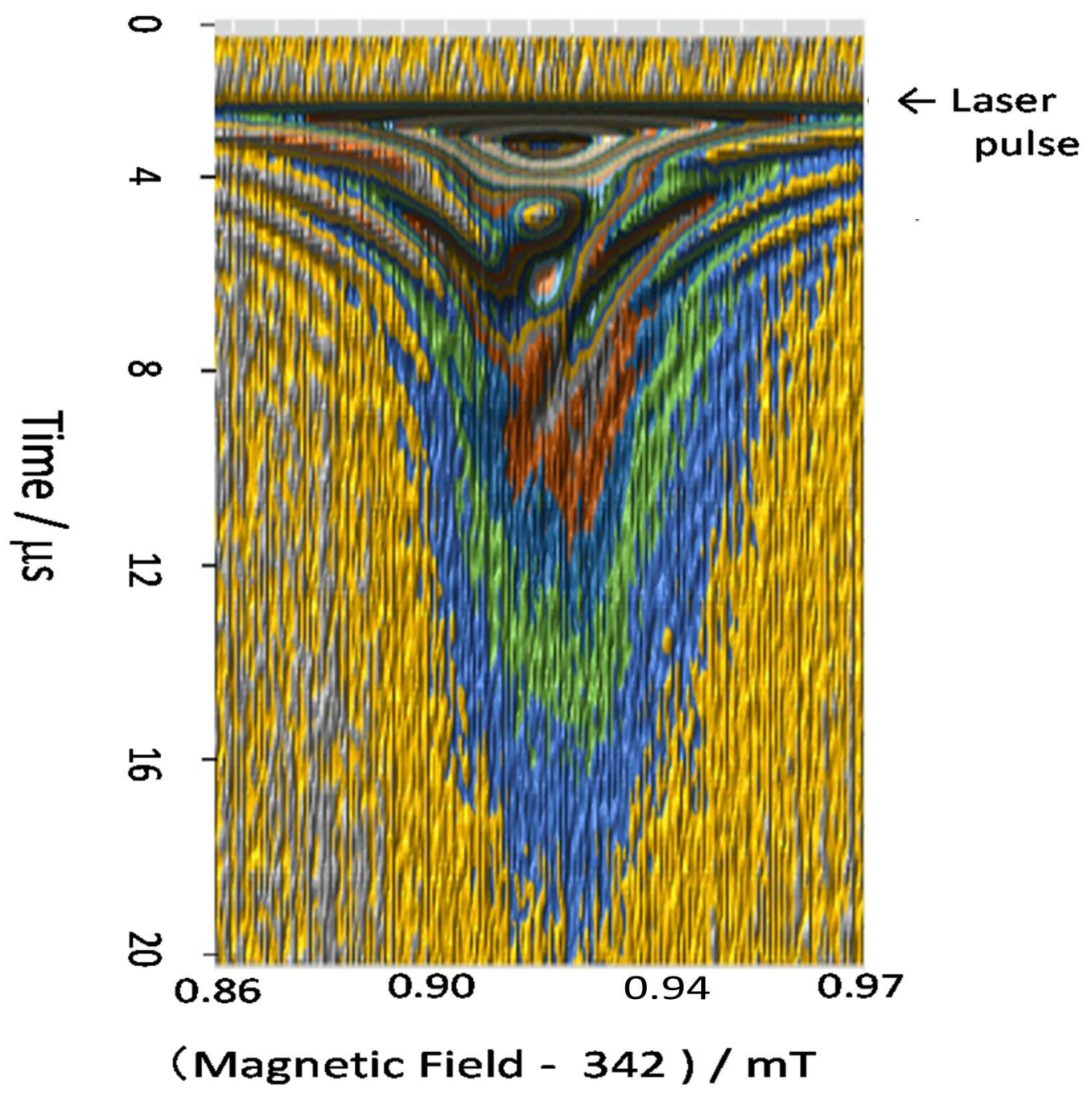

Fig S6 Two dimensional TR-ESR plot as a function of time and magnetic field for

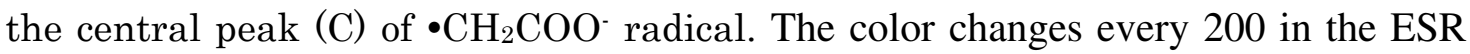
intensity. This is a top view of the 3D plot in Figure 9, and some cross sections at various time and some magnetic fields were shown in the main report as Figures 10 and 11, respectively. The time resolution was $0.16 \mu$ s and the magnetic field step was $0.000488 \mathrm{mT}$. 

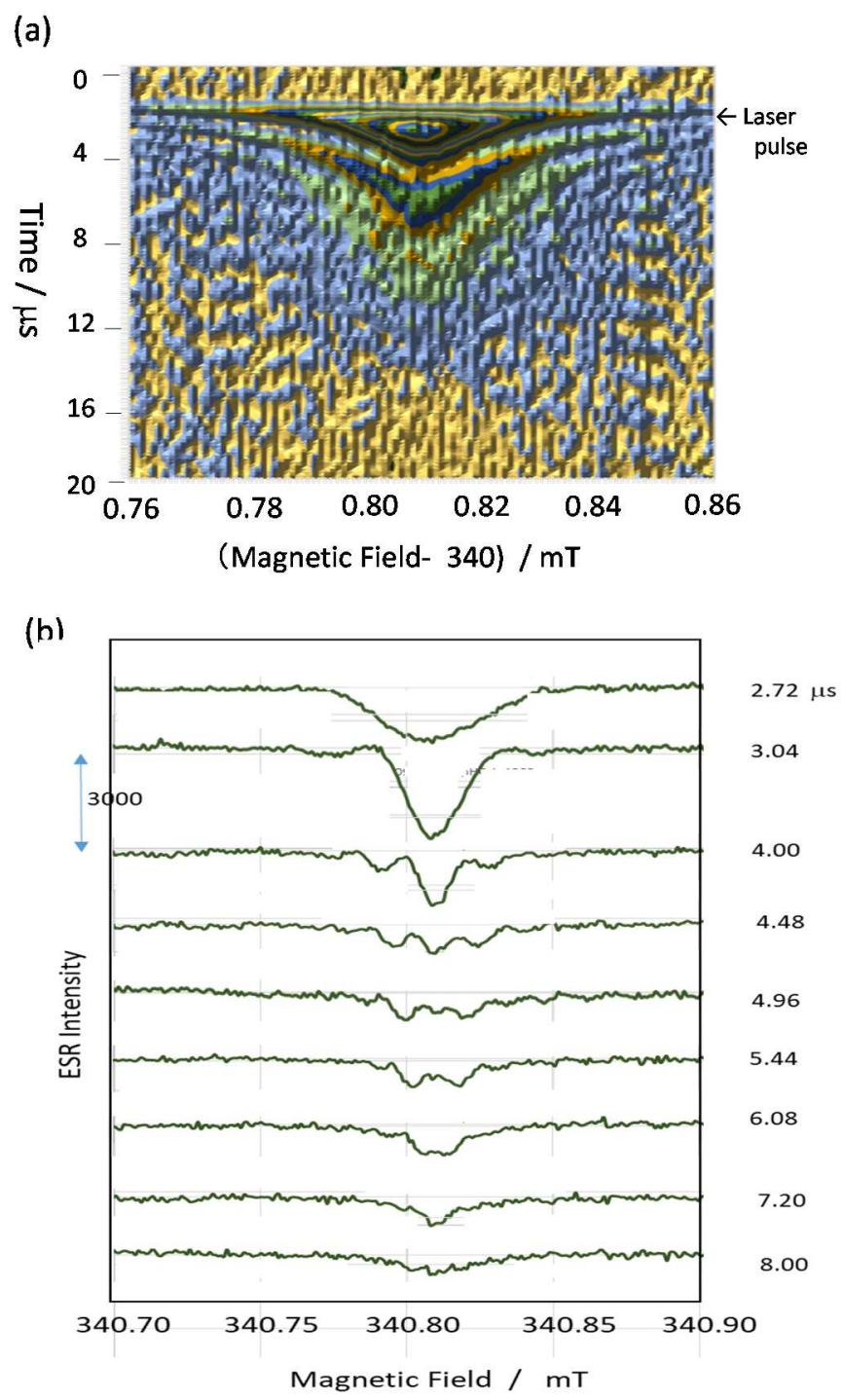

Fig S7 (a) Two dimensional TR-ESR plot as a function of time and magnetic field for the low field signal (L) of ${ }^{-} \mathrm{CH}_{2} \mathrm{COO}^{-}$radical. The color changes every 200 in the ESR intensity. (b) Cross sections of (a) at the various times indicated. The time resolution was $0.16 \mu$ s and the magnetic field step was $0.000977 \mathrm{mT}$. 


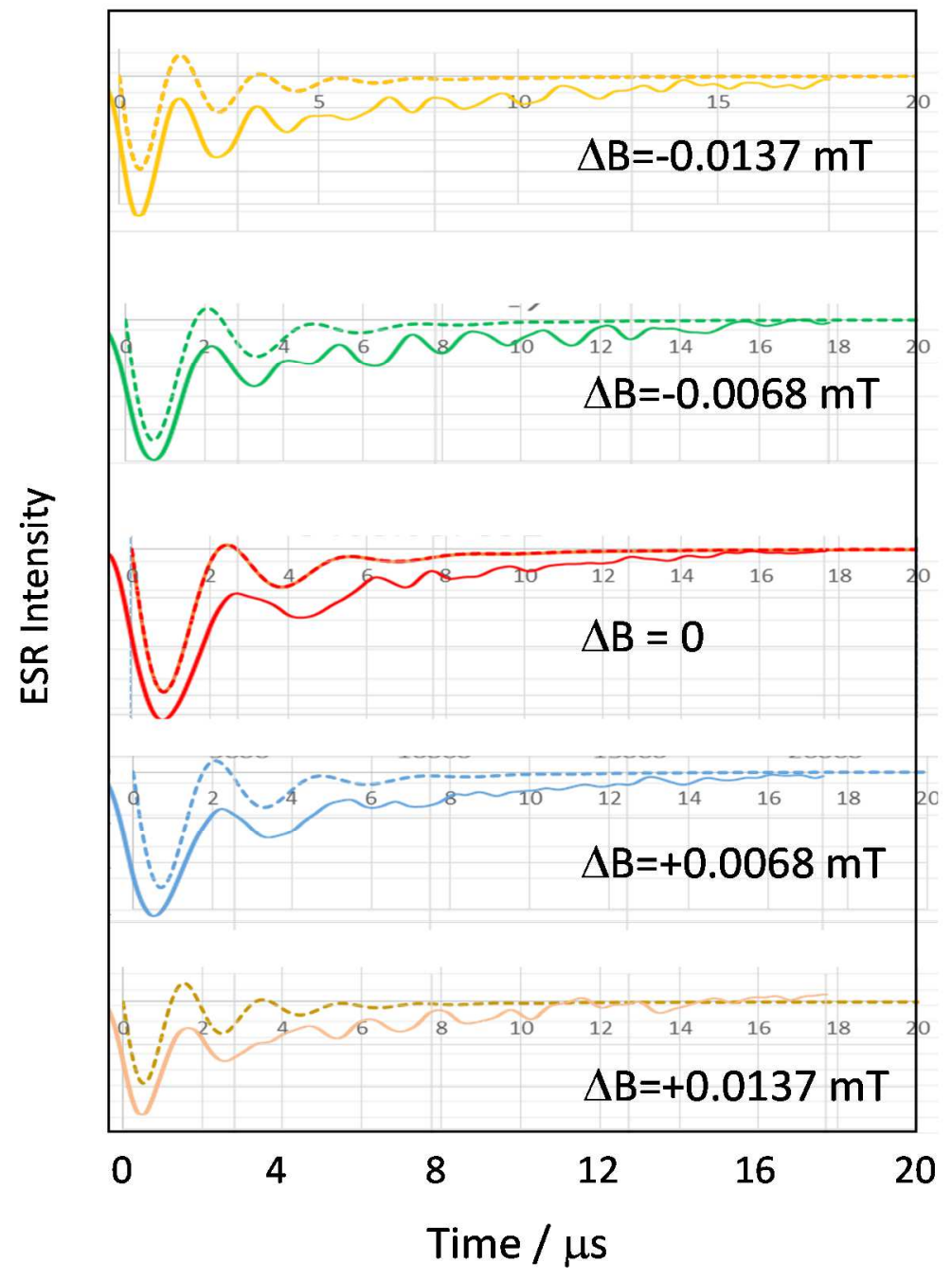

Fig S8 Time dependence of the low-field ESR signal (L) of $\cdot \mathrm{CH}_{2} \mathrm{COO}^{-}$at various magnetic fields as cross sections of Figure $S 7(\mathrm{a}) . \Delta \mathrm{B}$ represents the difference of the magnetic field from the resonant magnetic field. Dotted lines were calculated with eq (1) using the parameters in Table 1 and $\omega_{1}=2.06 \times 10^{6} \mathrm{~s}^{-1}$, where $\Delta \omega$ was calculated from $\Delta \mathrm{B}$ without any factor. 